\title{
Iterative Decoding for Noisy Network Coding for Two-Way Relay Channels
}

\author{
Michael Heindlmaier, Markus Staudacher (Jäger) \\ Institute for Communications Engineering, Technische Universität München, Munich, Germany \\ Email: michael.heindlmaier@tum.de, markus.jaeger@mytum.de
}

\begin{abstract}
This paper presents a practical implementation of Noisy Network Coding (NNC) for half-duplex Two-Way Relay channels (TWRC). With Noisy Network Coding, the relay quantizes the incoming signals and forwards them digitally. The receivers do not try to recover the relay quantization index but the codewords that are embedded in the quantized signal. Our approach uses irregular repeat-accumulate low-density parity check (LDPC) codes to implement NNC. We derive the joint factor graph and the message passing rules for the corresponding joint iterative decoding scheme. Our simulation results confirm the performance advantages predicted by random coding arguments.
\end{abstract}

\section{INTRODUCTION}

The Two-Way Relay Channel (TWRC) is a prominent example for cooperative relaying because it contains challenging subproblems such as Multiple Access (MAC) interference and Broadcast (BC) with side information. One of the possible coding schemes for the TWRC is Quantize-and-Forward (QF), where the relay quantizes its incoming signal and forwards this information digitally. Although other coding schemes can achieve higher performance, Quantize-and-Forward (QF) is an attractive choice because it does not require the relay to decode. Several schemes were introduced for $\mathrm{QF}$ and the TWRC, e.g. [1], [2], [3]. QF was extended to general Gaussian networks by Avestimehr et al. in [4] in a strategy called Quantize-Map-and-Forward (QMF). The scheme was further generalized by Yassaee et al. [5] and Lim et al. [6] for general multi-hop networks. This scheme is today known as Noisy Network Coding (NNC). The coding scheme in [3] can be seen as a particular derivation of Noisy Network Coding (NNC) for the TWRC. The achievability proofs of all the mentioned QF schemes involve random codes, so they cannot be directly used in practice. We bridge this gap by proposing an iterative decoding scheme derived in Section IV for NNC and the TWRC. For the Relay Channel, a decoder for QMF has been studied in [7] with Low Density Parity Check (LDPC) and Low Density Generator Matrix (LDGM) codes. For the diamond channel, an iterative message passing decoder for QMF has been proposed in [8], [9].

Our main contributions are to formulate the decoding problem of NNC for the TWRC on a factor graph, to derive the corresponding message passing rules and to provide simulation results of practical codes for this setup.

This paper is organized as follows: We explain the system model in Section II. Section III reviews several coding schemes. Section IV introduces the factor graph of the system and defines iterative decoding schemes on this graph. We plot performance results of the implemented system in Section V.

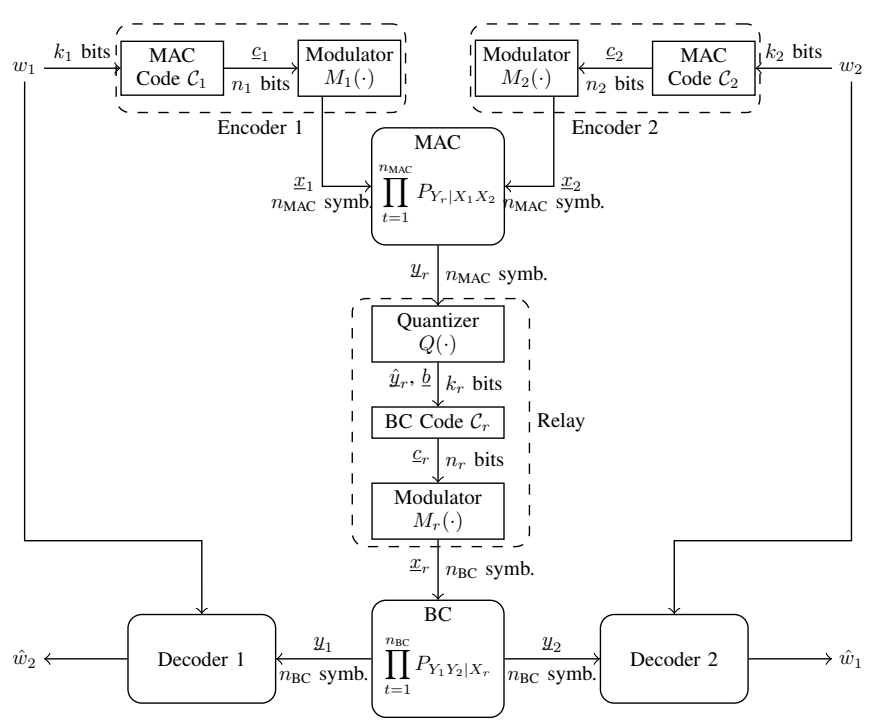

Fig. 1: Block diagram.

\section{System Model}

Fig. 1 depicts the system setup, as described below.

Notation: We write random variables with upper-case letters, e.g. $X$, their outcomes (values) by lower-case letters, e.g. $x$. Vectors are written with underlined letters, e.g. $\underline{x}$. The probability of $X$ taking on the value $x$ is written as $\bar{P}_{X}(x)$, where the subscript is sometimes omitted if clear from the context. The indicator function $\mathbb{1}\{\cdot\}$ takes on the value 1 if the event inside the brackets is true and 0 otherwise. Matrices are written boldface, e.g. $\mathbf{A} . \mathbf{A}^{\dagger}$ is the transpose of matrix $\mathbf{A}$.

Encoding at the Transceivers: The system has two transceivers $\mathrm{Rx}_{1}$ and $\mathrm{Rx}_{2}$, that exchange their independent messages $w_{1} \in\left\{1,2, \ldots, 2^{n R_{1}}\right\}, w_{2} \in\left\{1,2, \ldots, 2^{n R_{2}}\right\}$ in $n$ channel uses through a relay node $r . \mathrm{Rx}_{1}$ and $\mathrm{Rx}_{2}$ cannot hear each other, so communication is possible only through the relay. The communication is split into two phases: In the multiple access (MAC) phase with $n_{\mathrm{MAC}}<n$ channel uses, $\mathrm{Rx}_{1}$ maps its observed message $w_{1}$ of $k_{1}=n R_{1}$ bits with a binary code $\mathcal{C}_{1}$ to a codeword $\underline{c}_{1}$ of $n_{1}$ bits. The binary code $\mathcal{C}_{1}$ has channel coding rate $R_{1}^{\mathrm{CC}}=\frac{k_{1}}{n_{1}}$. The modulator function maps the binary codeword to the channel input vector $\underline{x}_{1}=M_{1}\left(\underline{c}_{1}\right)$ of $n_{\mathrm{MAC}}$ symbols. In particular, with slight abuse of notation, a single symbol is obtained by $x_{1, t}=M_{1}\left(\underline{c}_{1, t}\right)$, where the vector $\underline{c}_{1, t}$ collects the $n_{1} / n_{\mathrm{MAC}}=R_{1}^{\mathrm{Mod}}$ bits determining the $t$-th symbol $x_{1, t} \in \mathcal{X}_{1} . \mathcal{X}_{1}$ represents the modulation alphabet of $\operatorname{Rx}_{1}$ and define $R_{1}^{\mathrm{Mod}}=\log _{2}\left(\left|\mathcal{X}_{1}\right|\right)$. Similarly for $\mathrm{Rx}_{2}$. The mapping from $w_{1}, w_{2}$ to $\underline{x}_{1}, \underline{x}_{2}$, 
respectively, is called the MAC code. Define $\alpha=n_{\mathrm{MAC}} / n$ as the time fraction of this first phase. The overall spectral efficiency (in terms of number of information bits for each of the $n$ time slots) of the MAC code at $\mathrm{Rx}_{j}$ is given by $R_{j}=\alpha \cdot R_{j}^{\mathrm{Mod}} R_{j}^{\mathrm{CC}}$.

Uplink (Multiple Access) Channel: The relay receives the output of the discrete memoryless channel that is governed by the distribution $P_{Y_{r} \mid X_{1} X_{2}}$. In all our numerical examples, we use the following model: The relay receives the superposition of the two channel inputs corrupted by additive white Gaussian noise $Z_{r}$ :

$$
y_{r, t}=x_{1, t}+x_{2, t}+z_{r, t}, \quad t=1,2, \ldots, n_{\mathrm{MAC}},
$$

where $Z_{r, t} \sim \mathcal{N}\left(0, N_{r}\right)$ and $\mathbb{E}\left[X_{1, t}^{2}\right] \leq P_{1}, \mathbb{E}\left[X_{2, t}^{2}\right] \leq P_{2}$. We use a high-rate scalar pre-quantization of $Y_{r}$ to make this model match the discrete memoryless model.

Quantization at the Relay: We focus on scalar quantization in this work, a possibly suboptimal but practical choice: The scalar quantizer function $Q: \mathcal{Y}_{r} \rightarrow \hat{\mathcal{Y}}_{r}$ maps each received value $y_{r, t}$ to a representative $\hat{y}_{r, t}$ from the finite symbol alphabet $\hat{\mathcal{Y}}_{r}$. The quantizer output bit sequence $\underline{b}_{t}$ for $y_{r, t}$ at the input is given by the mapping $\beta: \mathcal{Y}_{r} \rightarrow \mathbb{F}_{2}^{R_{r}^{\text {Quant }}}$, where $R_{r}^{\text {Quant }}=\log _{2}\left(\left|\hat{\mathcal{Y}}_{r}\right|\right)$ denotes the number of quantizer output bits per input symbol. Hence,

$$
\hat{y}_{r, t}=Q\left(y_{r, t}\right), \quad \underline{b}_{t}=\beta\left(y_{r, t}\right) .
$$

The individual bits in the block $\underline{b}_{t}$ may be (and generally are) correlated. The quantizer outputs $k_{r} \triangleq n_{\mathrm{MAC}} R_{r}^{\text {Quant }}$ bits in total. With slight abuse of notation we denote the whole quantized representation by $\hat{y}_{r}=Q\left(y_{r}\right)$ and the corresponding bit sequence by $\underline{b}=\beta\left(\underline{y}_{r}\right)$.

Encoding at the Relay: During the Broadcast $(\mathrm{BC})$ phase with $n_{\mathrm{BC}}=n-n_{\mathrm{MAC}}=(1-\alpha) n$ channel uses, the relay transmits the codeword $\underline{x}_{r}(\underline{b})$ corresponding to the quantizer bit sequence $\underline{b}=\beta\left(\underline{y}_{r}\right)$. The mapping from $\underline{b}$ to $\underline{x}_{r}$ is found by a Joint Source Channel Code (JSCC) (see e.g. [10]): The binary quantizer output $\underline{b}$ is mapped ${ }^{1}$ to a $n_{r}$-bit codeword $\underline{c}_{r}$ of the code $\mathcal{C}_{r}$. The binary code $\mathcal{C}_{r}$ has a code rate $R_{r}^{\mathrm{JSCC}}=\frac{k_{r}}{n_{r}}$.

The relay modulator maps this codeword to the channel input $\underline{x}_{r}=M_{r}\left(\underline{c}_{r}\right)$. Again, we refer to a single channel input symbol by $x_{r, t}=M_{r}\left(\underline{c}_{r, t}\right)$, where $\underline{c}_{r, t}$ collects the $n_{r} / n_{\mathrm{BC}}=$ $R_{r}^{\text {Mod }}$ bits determining the $t$-th broadcast transmit symbol. The mapping from $\underline{b}$ to $\underline{x}_{r}$ is called the $B C$ code.

Downlink (Broadcast) Channel: The received signals at $\mathrm{Rx}_{1}$ and $\mathrm{Rx}_{2}$ are:

$$
y_{j, t}=x_{r, t}+z_{j, t}, \quad t=1, \ldots, n_{\mathrm{BC}}
$$

for $j \in\{1,2\}, \mathbb{E}\left[X_{r, t}^{2}\right] \leq P_{r}$ and $Z_{j, t} \sim \mathcal{N}\left(0, N_{j}\right)$.

Decoding at the Transceivers: $\mathrm{Rx}_{2}$ computes an estimate $\hat{w}_{1}$ of $w_{1}$ by using the received signal $y_{2}$ and its own message $w_{2}$ as side information. $\mathbf{R x}_{1}$ operates similarly.

\footnotetext{
${ }^{1}$ As the quantizer output $\underline{b}$ is correlated, one can compress these $k_{r}=$ $n_{\mathrm{MAC}} R_{r}^{\text {Quant }}$ bits to $n_{\mathrm{MAC}} R_{Q}$ bits, with a source coding rate $R_{r}^{\mathrm{SC}}=\frac{R_{r}^{\text {Quant }}}{R_{Q}} \geq$ 1 . One can then map these $n_{\mathrm{MAC}} R_{Q}$ bits to an $n_{r}$-bit codeword $\underline{c}_{r}$, with channel coding rate $R_{r}^{\mathrm{CC}}=\frac{n_{\mathrm{MAC}} R_{Q}}{n_{r}}$. The overall joint source channel coding rate is given by $R_{r}^{\mathrm{JSCC}}=\frac{k_{r}}{n_{r}}$
}

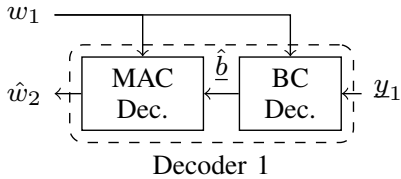

(a) Decoder for $\mathcal{R}_{\mathrm{CF}}$.

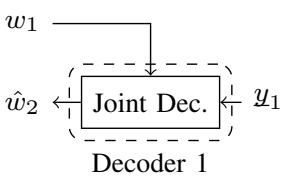

(b) Decoder for $\mathcal{R}_{\mathrm{NNC}}$.
Fig. 2: Decoder structures.

\section{PERFORMANCE LIMITS}

We briefly summarize the coding schemes to be compared in Section V. Due to space limitations we omit rate plots and refer to [11, Chapter 2].

\section{A. Compress-and-Forward (CF)}

Schnurr et. al. [2] and Kim et. al. [12] derived an achievable rate region using Tuncel's results for Slepian-Wolf coding over broadcast channels [13]. The achievable rate region is the set $\mathcal{R}_{\mathrm{CF}} \subset \mathbb{R}_{+}^{2}$ of rate tuples $\left(R_{1}, R_{2}\right)$ satisfying

$$
\begin{aligned}
R_{1} \leq \alpha I\left(X_{1} ; \hat{Y}_{r} \mid X_{2} U\right), \quad R_{2} & \leq \alpha I\left(X_{2} ; \hat{Y}_{r} \mid X_{1} U\right) \\
\text { subject to } \quad \alpha I\left(Y_{r} ; \hat{Y}_{r} \mid X_{2} U\right) & \leq(1-\alpha) I\left(X_{r} ; Y_{2}\right) \\
\alpha I\left(Y_{r} ; \hat{Y}_{r} \mid X_{1} U\right) & \leq(1-\alpha) I\left(X_{r} ; Y_{1}\right)
\end{aligned}
$$

for some $P_{U} P_{X_{1} \mid U} P_{X_{2} \mid U} P_{Y_{r} \mid X_{1} X_{2}} P_{\hat{Y}_{r} \mid Y_{r} U}$ and $P_{X_{r}} P_{Y_{1} Y_{2} \mid X_{r}}$, $\alpha>0,|\mathcal{U}| \leq 4,\left|\hat{\mathcal{Y}}_{r}\right| \leq\left|\mathcal{Y}_{r}\right|+3$.

Decoder Structure: Two decoding steps are required at each receiver: The coding scheme requires reliable decoding of $\underline{b}$ at the receiver. The $\mathrm{BC}$ code is decoded using its own message as a priori knowledge. Knowing $\underline{b}$, the desired message is decoded, again using its own message as side information. The decoder structure is shown in Fig. 2a.

\section{B. Noisy Network Coding / Joint Decoding (NNC)}

Schnurr et al. derived an achievable rate region in [3] that employs a single decoding stage. Unlike $\mathrm{CF}$, it does not require reliable decoding of the quantizer output $\underline{b}$ but looks only for the intended message in a joint decoder. In the literature, this is called simultaneous non-unique decoding [14], because the decoder might return the correct message of interest, but a wrong quantization index. The achievable rate region is given by the set $\mathcal{R}_{\mathrm{NNC}} \subset \mathbb{R}_{+}^{2}$ of rate tuples $\left(R_{1}, R_{2}\right)$ satisfying

$$
\begin{aligned}
& R_{1} \leq \alpha I\left(X_{1} ; \hat{Y}_{r} \mid X_{2} U\right) \\
& R_{1} \leq(1-\alpha) I\left(X_{r} ; Y_{2}\right)-\alpha I\left(Y_{r} ; \hat{Y}_{r} \mid X_{1} X_{2} U\right) \\
& R_{2} \leq \alpha I\left(X_{2} ; \hat{Y}_{r} \mid X_{1} U\right) \\
& R_{2} \leq(1-\alpha) I\left(X_{r} ; Y_{1}\right)-\alpha I\left(Y_{r} ; \hat{Y}_{r} \mid X_{1} X_{2} U\right)
\end{aligned}
$$

for some $P_{U} P_{X_{1} \mid U} P_{X_{2} \mid U} P_{Y_{r} \mid X_{1} X_{2}} P_{\hat{Y}_{r} \mid Y_{r}}$ and $P_{X_{r}} P_{Y_{1} Y_{2} \mid X_{r}}$ and $\alpha>0$. It suffices to consider $|\mathcal{U}| \leq 3,\left|\hat{\mathcal{Y}}_{r}\right| \leq\left|\mathcal{Y}_{r}\right|+2$.

Decoder Structure: the decoder must jointly decode the $\mathrm{BC}$ code and the MAC code in a single-stage decoder using its own message as side information. $\underline{b}$ is not required to be decoded (correctly). The decoder structure is shown in Fig. 2 b. 


\section{ITERATIVE DECODING}

The decoding of both $\mathrm{CF}$ and $\mathrm{NNC}$ can be explained on the same factor graph that will be described in this section. The schemes differ in the exact decoding operations on this graph that are explained in more detail in Section IV-C.

The efficient operation of QF schemes requires multiple quantization levels and higher-order modulation in the downlink. To accommodate binary codes, we follow the design paradigm of Bit-Interleaved Coded Modulation (BICM) [15].

\section{A. Coding and Modulation}

For $\mathcal{C}_{1}$ we use a linear code specified by the sparse parity check matrix $\mathbf{H}_{1} \in \mathbb{F}_{2}^{n_{1}-k_{1} \times n_{1}}$ where all codewords $\underline{c}_{1} \in \mathcal{C}_{1}$ satisfy $\mathbf{H}_{1} \underline{c}_{1}^{\dagger}=\underline{0} . \underline{c}_{1}^{\dagger}$ denotes the transpose of the row vector $\underline{c}_{1}$. The parity check matrix can be represented by a bipartite graph. The $i$-th parity check node, $i \in\left\{1, \ldots, n_{1}-k_{1}\right\}$, is connected to all variable nodes indexed by the set $\mathcal{S}_{i}^{\mathcal{C}_{1}}$. The $i$-th parity check equation thus reads as $\sum_{j \in \mathcal{S}_{i}^{\mathcal{C}_{1}}} c_{1, j}=0$. We will use LDPC codes for $\mathcal{C}_{1}$ and $\mathcal{C}_{2}$.

At the relay, we use a linear joint source-channel encoder that maps the $k_{r}$-bit quantizer output sequence $b$ to the codeword $\underline{c}_{r}$ of length $n_{r}$. We can write $\underline{c}_{r}=\underline{b} \mathbf{G}_{r}$, with $\mathbf{G}_{r} \in \mathbb{F}_{2}^{k_{r}} \bar{x}_{r}$ being the generator matrix relating quantizer output and relay codeword. $\underline{b}$ and $\underline{c}_{r}$ are row vectors. We use systematic Irregular Repeat-Accumulate (IRA) codes [16], [17] for this task, hence the codeword $\underline{c}_{r} \in \mathcal{C}_{r}$ can be written as $\underline{c}_{r}=\left[\underline{b}_{\pi}, \underline{p}_{\text {IRA }}\right]$, where $\underline{b}_{\pi}$ is a permuted version of the information sequence $\underline{b}$ and $\underline{\underline{I R A}}_{\mathrm{IR}}$ denotes the parity bits formed by the systematic code. The $i$-th encoding equation, $i \in\left\{1, \ldots, n_{r}\right\}$ involves a subset of the bits of $\underline{b}$, indexed by $\mathcal{S}_{i}^{\mathcal{C}_{r}}$, such that $c_{r, i}=\sum_{k \in \mathcal{S}_{i}^{\mathcal{C}_{r}}} b_{k}$.

The bit labeling of the different constellation symbols of all modulators will be binary reflected Gray mapping [18], unless stated differently. As we separate binary coding and modulation at both encoder and decoder, this is a BICM approach.

\section{B. Factor Graph}

The factor graph is a graphical representation of the joint probability distribution

$P\left(\underline{c}_{1}, \underline{c}_{2}, \underline{x}_{1}, \underline{x}_{2}, \underline{y}_{r}, \underline{b}_{,} \underline{c}_{r}, \underline{x}_{r}, \underline{y}_{1}, \underline{y}_{2}\right)=P\left(\underline{c}_{1}\right) P\left(\underline{c}_{2}\right) P\left(\underline{x}_{1} \mid \underline{c}_{1}\right)$. $P\left(\underline{x}_{2} \mid \underline{c}_{2}\right) P\left(\underline{y}_{r} \mid \underline{x}_{1}, \underline{x}_{2}\right) P\left(\underline{b}_{\mid} \underline{y}_{r}\right) P\left(\underline{c}_{r} \mid \underline{b}_{)}\right) P\left(\underline{x}_{r} \mid \underline{c}_{r}\right) P\left(\underline{y}_{1}, \underline{y}_{2} \mid \underline{x}_{r}\right)$.

The conditional distributions can be further factorized as

$$
\begin{aligned}
P\left(\underline{y}_{2} \mid \underline{x}_{r}\right) & =\prod_{t=1}^{n_{\mathrm{BC}}} P_{Y_{2} \mid X_{r}}\left(y_{2, t} \mid x_{r, t}\right) \\
P\left(\underline{x}_{r} \mid \underline{c}_{r}\right) & =\mathbb{1}\left\{\underline{x}_{r}=M_{r}\left(\underline{c}_{r}\right)\right\}=\prod_{t=1}^{n_{\mathrm{BC}}} \mathbb{1}\left\{x_{r, t}=M_{r}\left(\underline{c}_{r, t}\right)\right\} \\
P\left(\underline{c}_{r} \mid \underline{b}\right) & =\mathbb{1}\left\{\underline{c}_{r}=\underline{b}_{r}\right\}=\prod_{i=1}^{n_{r}} \mathbb{1}\left\{c_{r, i}=\sum_{k \in \mathcal{S}_{i}^{\mathcal{C}_{r}}} b_{k}\right\} \\
P\left(\underline{b} \mid \underline{y}_{r}\right) & =\mathbb{1}\left\{\underline{b}=\beta\left(\iota\left(\underline{y}_{r}\right)\right)\right\}=\prod_{t=1}^{n_{\mathrm{MAC}}} \mathbb{1}\left\{\underline{b}_{t}=\beta\left(\iota\left(y_{r, t}\right)\right)\right\} \\
P\left(\underline{y}_{r} \mid \underline{x}_{1}, \underline{x}_{2}\right) & =\prod_{t=1}^{n_{\mathrm{MAC}}} P_{Y_{r} \mid X_{1} X_{2}}\left(y_{r, t} \mid x_{1, t}, x_{2, t}\right) \\
P\left(\underline{x}_{1} \mid \underline{c}_{1}\right) & =\mathbb{1}\left\{\underline{x}_{1}=M_{1}\left(\underline{c}_{1}\right)\right\}=\prod_{t=1}^{n_{\mathrm{MAC}}} \mathbb{1}\left\{x_{1, t}=M_{1}\left(\underline{c}_{1, t}\right)\right\}
\end{aligned}
$$

$$
P\left(\underline{c}_{1}\right) \propto \mathbb{1}\left\{\underline{c}_{1} \in \mathcal{C}_{1}\right\}=\prod_{i=1}^{k_{1}-n_{1}} \mathbb{1}\left\{\sum_{k \in \mathcal{S}_{i}^{\mathcal{C}_{1}}} c_{1, k}=0\right\} .
$$

A simplified factor graph is shown in Fig. 3 from the perspective of $\mathrm{Rx}_{2}$. $\mathrm{Rx}_{2}$ knows its own transmitted signal $\underline{x}_{2}$ and observes the received signal $y_{2}$. These two sequences serve as inputs for the decoder operating on the factor graph. The MAC code of $\mathrm{Rx}_{1}$ with codeword $\underline{c}_{1} \in \mathcal{C}_{1}$ is depicted on the top of the graph. The parity check matrix $\mathbf{H}_{1}$ is represented by the interleaver $\Pi^{\mathcal{C}_{1}}$ connecting code bit variable nodes and parity check nodes. The codeword $\underline{c}_{1}$ is unknown to $\mathrm{Rx}_{2}$ and should be decoded. The interleaver $\Pi_{\underline{b}}^{\mathcal{C}_{r}}$ relates $\underline{b}$ and $\underline{b}_{\pi}$, the interleaver $\Pi^{\mathrm{IRA}}$ defines the accumulator. The factor graph in Fig. 3 is simplified with regard to two aspects:

1: For scalar quantizers one can describe the modulator of $\mathrm{Rx}_{1}$, the uplink channel and the quantizer by a single special factor node that connects $\underline{c}_{1, t}, x_{2, t}$ and $\underline{b}_{t}$ for all $t$, with factor node kernel function $P\left(\underline{b}_{t} \mid \underline{c}_{1, t}, x_{2, t}\right)$. This is possible because of the factorization

$$
P\left(\underline{b}_{1} \underline{c}_{1}, \underline{x}_{2}\right)=\prod_{t=1}^{n_{\mathrm{MAC}}} P\left(\underline{b}_{t} \mid \underline{c}_{1, t}, x_{2, t}\right) .
$$

2: If we use bit-metric inputs to the decoder of the $\mathrm{BC}$ code and no not perform iterative demapping and decoding as in [19], we can account for the relay modulator, channel input and downlink channel as at the bottom in Fig. 3: Each code bit of $\underline{c}_{r}$ obtains a log-likelihood value from the channel observation $\stackrel{-}{L}\left(c_{r, t} \mid \underline{y}_{2}\right)$ that is kept constant during the decoding process. The performance loss due to this simplification is small [15] for a modulator applying binary reflected Gray mapping.

\section{Decoding}

Compress \& Forward: As shown in Fig. 2a, one must first decode $\underline{b}$ with the observation $y_{2}$, but also taking into account the correlation of $\underline{b}$ with $\underline{x}_{2}$. This results in bit-wise a-priori LLRs formed according to the distribution $P_{\underline{B}_{t} \mid X_{2}}$ (corresponding to $P_{\hat{Y}_{r} \mid X_{2}}$ ). One can interpret this as removing the edges from the special nodes to $\underline{c}_{1}$ in Fig. 3 in the first decoding step. The BC decoder output $\hat{b}$ is used to form $L\left(c_{1, k} \mid \underline{b}\right)$ for the input of the MAC decoder.

Noisy Network Coding: For $\mathrm{NNC}, \mathrm{Rx}_{2}$ tries to iteratively decode $\underline{c}_{1}$ using the whole graphical structure. The only inputs are $\underline{x}_{2}$ and $\underline{y}_{2}$. The decoder cares only about correct decoding of $\underline{c}_{1}$ and neglects bit errors in $\underline{b}$.

The typical Belief Propagation (BP) decoder usually resorts to bit-wise Maximum A-Posteriori (MAP)-decoding [20, Chapter 2.5]. The rule for bit-wise MAP-decoding for the $k$-th bit of $\underline{c}_{1}$ is given by

$\hat{c}_{1, k}^{\mathrm{MAP}}=\underset{c_{1, k} \in\{0,1\}}{\operatorname{argmax}} P\left(c_{1, k} \mid \underline{x}_{2}, \underline{y}_{2}\right)=\underset{c_{1, k} \in\{0,1\}}{\operatorname{argmax}} \sum_{\underline{c}_{1} \sim c_{1, k}} P\left(\underline{c}_{1} \mid \underline{x}_{2}, \underline{y}_{2}\right)$

where $\sum_{\underline{c}_{1} \sim c_{1, k}}$ indicates a summation over all components of the vector $\underline{c}_{1}$ except $c_{1, k}$.

Note that the factor graph contains cycles and thus the sumproduct computation will not necessarily lead to the correct result. However, it usually suffices for the graph to be locally tree-like to achieve a good decoding performance. 


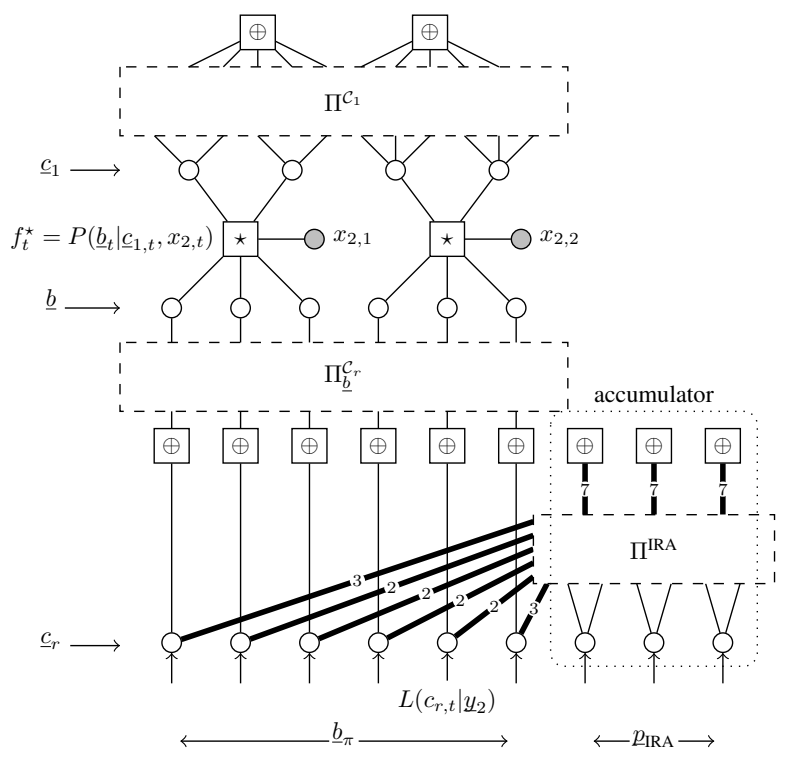

Fig. 3: Simplified overall factor graph from the perspective of $\mathrm{Rx}_{2}$, with specific implementation choices. In this example, we have $R_{1}^{\text {Mod }}=2\left(2 \mathrm{bits} /\right.$ symbol for $\left.\mathrm{Rx}_{1}\right), n_{1}=4, R_{1}^{\mathrm{CC}}=\frac{1}{2}, n_{\mathrm{MAC}}=2$, $R_{r}^{\text {Quant }}=3, R_{r}^{\mathrm{JSCC}}=\frac{2}{3}$. A systematic Irregular Repeat-Accumulate (IRA) code is used as the joint source channel code at the relay. Bold edges represent multiple edges, with the number of single edges indicated by the corresponding number. The information of the received sequence $\underline{y}_{2}$ is mapped to individual bit-metric likelihood values $L\left(c_{r, t} \mid y_{2}\right)$ for each relay code bit $c_{r, t}$. Iterative demapping at the demodulator nodes is not employed. From the figure, the modulation format at the relay cannot be deduced. If we assume $R_{r}^{\mathrm{Mod}}=3$ (3 bits/symbol), then $n_{\mathrm{BC}}=3$ and hence $\alpha=\frac{2}{5}$.

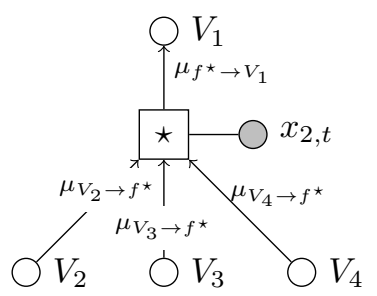

Fig. 4: Special factor node in abstract form.

\section{Message Passing Rules}

Each edge carries one message $\mu(v)$ for each possible value $v$ of the connected variable node $V$. The message updates at the regular variable and check nodes are the standard updates as in derived in [20]. The special factor node has a different update rule: As shown in Fig. 3 and Fig. 4, the special factor node is connected to a fixed number of variable nodes that depends on the modulation format rate $R_{1}^{\mathrm{Mod}}$ and the quantizer rate $R_{r}^{\text {Quant }}$. The $t$-th special factor node is connected to $\underline{c}_{1, t}$, $\underline{b}_{t}$ and $x_{2, t}$, where the value of $x_{2, t}$ is known. To simplify notation, we stack $\underline{c}_{1, t}$ and $\underline{b}_{t}$ into the vector $\underline{v}_{t} \triangleq\left[\underline{c}_{1, t}, \underline{b}_{t}\right]$. $v_{t, i}$ corresponds to the $i$-th entry of the vector $\underline{v}_{t}$, hence it corresponds to a bit of either $\underline{c}_{1, t}$ or $\underline{b}$. Define the special factor node kernel function as

$$
f_{t}^{\star}\left(\underline{v}_{t}, x_{2, t}\right)=P\left(\underline{b}_{t} \mid \underline{c}_{1, t}, x_{2, t}\right) .
$$

For a special factor node $f_{t}^{\star}$ with connected variable nodes indexed by $\mathcal{S}_{t}^{\star}$ (written as $\underline{v}_{\mathcal{S}_{t}^{\star}}$ ), one obtains the message update rule [21] $\forall v_{j} \in\{0,1\}$ :

$$
\mu_{f_{t}^{\star} \rightarrow V_{j}}\left(v_{j}\right)=\sum_{v_{i} \in\{0,1\}, i \in \mathcal{S}_{t}^{\star} \backslash\{j\}} f_{t}^{\star}\left(\underline{v}_{\mathcal{S}_{t}^{\star}}, x_{2, t}\right) \prod_{i \in \mathcal{S}_{t}^{\star} \backslash\{j\}} \mu_{v_{i} \rightarrow f_{t}^{\star}}\left(v_{i}\right)
$$

For example, let $R_{1}^{\text {Mod }}=2, R_{r}^{\text {Quant }}=2$, as in Fig. 4 and let $\underline{c}_{1, t}=\left[c_{1,1} c_{1,2}\right], \underline{b}_{t}=\left[b_{1} b_{2}\right]$. Suppose $V_{j}$ corresponds to the most significant bit in the constellation label $\underline{c}_{1, t}$, hence $v_{j} \triangleq c_{1,1}$. The update of (9) in this case is given by

$$
\begin{gathered}
\mu_{f_{t}^{\star} \rightarrow C_{1,1}}\left(c_{1,1}=0\right)=\sum_{c_{1,2}, b_{1}, b_{2} \in\{0,1\}} P\left(b_{1} b_{2} \mid c_{1,1}=0, c_{1,2}, x_{2, t}\right) . \\
\mu_{C_{1,2} \rightarrow f_{t}^{\star}}\left(c_{1,2}\right) \cdot \mu_{B_{1} \rightarrow f_{t}^{\star}}\left(b_{1}\right) \cdot \mu_{B_{2} \rightarrow f_{t}^{\star}}\left(b_{2}\right)
\end{gathered}
$$

and accordingly for the message for $c_{1,1}=1$.

\section{Numerical Results}

We focus on one-dimensional modulation schemes to reduce computational complexity for simulations. All results apply to complex signaling as well.

We consider a symmetric scenario for our simulations, i.e. $P_{1}=P_{2}$ and $N_{1}=N_{2}$ and let the downlink have an SNR offset of $6 \mathrm{~dB}$. In this case both users have the same achievable rate, which depends on the time-sharing fraction $\alpha$ and the quantizer. The optimal quantizer and time fraction depend on the SNR. Though suboptimal, we choose a Lloyd-Max (LM) quantizer ${ }^{2}$. Methods to optimize quantizers are derived in [11]. For our simulations it is convenient to use a constant time-sharing fraction. Varying fractions require varying block lengths for the channel codes which makes a fair comparison difficult. We use channel codes with rate $R_{1}^{\mathrm{CC}}=R_{2}^{\mathrm{CC}}=\frac{1}{2}$ for both transceivers and set $\alpha=\frac{1}{3}$. Hence we obtain a sum rate of $2 \cdot \frac{1}{2} \cdot \frac{1}{3}=\frac{1}{3}$ With these parameters, the required $\mathrm{SNR}=\frac{P_{1}}{N_{r}}$ is $1.1 \mathrm{~dB}$ for $\mathrm{NNC}$ and $1.5 \mathrm{~dB}$ for $\mathrm{CF}$.

Code Parameters: For the bit error curves, both transceivers use the WiMAX Irregular Repeat-Accumulate (IRA) LDPC code with code rate $R_{1}^{\mathrm{CC}}=R_{2}^{\mathrm{CC}}=\frac{1}{2}$ for $\mathcal{C}_{1}, \mathcal{C}_{2}$, respectively. The block length is $n_{1}=n_{2}=1152$, hence $k_{1}=k_{2}=576$. The normalized degree distribution from node perspective is $0.4583 x^{2}+0.3333 x^{3}+0.2084 x^{6}$ for the variable nodes, and $\frac{2}{3} x^{6}+\frac{1}{3} x^{7}$ for the check nodes. $\mathrm{Rx}_{1}$ and $\mathrm{Rx}_{2}$ use BPSK modulation, hence $R_{1}^{\mathrm{Mod}}=R_{2}^{\mathrm{Mod}}=1$ and $n_{\mathrm{MAC}}=1152$. The relay quantizes its signal with $R_{r}^{\text {Quant }}=3$ bits per received symbol, hence the Lloyd-Max quantizer output $\underline{b}$ consists of $k_{r}=3 \cdot n_{\mathrm{MAC}}=3456$ bits. The joint source channel code is a systematic IRA LDPC code $\mathcal{C}_{r}$ with rate $R_{r}^{\mathrm{JSCC}}=\frac{1}{2}$, hence $n_{r}=2 \cdot k_{r}=6912$. The normalized degree distribution from node perspective is $0.0613 x^{2}+0.6325 x^{3}+0.3062 x^{13}$ for the variable nodes, and $x^{6}$ for the check nodes. The relay uses 8ASK as modulation format, hence $R_{r}^{\mathrm{Mod}}=3$ and $n_{\mathrm{BC}}=\frac{n_{r}}{3}=2304$. As mentioned, this leads to $\alpha=\frac{1152}{1152+2304}=\frac{1}{3}$.

\footnotetext{
${ }^{2}$ The Lloyd-Max (LM) quantizer has contiguous quantization regions which is advantageous for a Gray bit labeling of $\underline{b}_{t}$.
} 
Bit Error Curves Whole System: Fig. 5 shows the performance of the whole system, i.e. combined uplink and downlink. For $\mathrm{CF}$, the estimated quantizer sequence $\hat{b}$ that has been decoded by the $\mathrm{BC}$ decoder is used to compute $\hat{w}_{1}$. The performance depends on the bit labeling of the quantization indices: Recall that the LM quantizer leads to contiguous quantization regions, hence each index has two distinct neighboring Voronoi regions (This cannot be guaranteed if the quantizer has noncontiguous quantization regions). We assign Gray labels to the quantization indices. If one bit is flipped in $\underline{b}$, we decide for a neighboring quantization region. This approach minimizes the resulting error of the LLR input to the MAC decoder. For comparison, the curves in Fig. 5b also show the PER for the $\mathrm{BC}$ code only. Although the BC code suffers from an error floor for $\mathrm{CF}$, this does not have an impact on the overall decoder. Even of there are some bit errors in $\hat{b}$, the MAC code is strong enough to decode $\hat{w}_{1}$ correctly. As expected, NNC achieves the better performance. For comparison, we also plot the error curves if we use a natural labeling of the quantization regions. We observe that the BER and PER curves have approximately the same distance to each other as the respective theoretical limits. Possible reasons for the gap to the theoretical limits are the moderate code length and the use of off-the-shelf codes. Optimization of codes like in [7] is a promising direction for improvement.

Remark 1: Note that the different performance curves are only due to different decoding algorithms. All schemes use the identical codes and hence the identical factor graph. The only difference is the LLR computation and the way the message passing decoder operates on this factor graph.

\section{CONCLUSION}

This paper presents implementation aspects of Noisy Network Coding for half-duplex Two-Way Relay Channels. We derive the joint factor graph, the corresponding decoder message passing rules and propose practical design choices. Finding optimized code ensembles for the factor graph structure is a worthwhile challenge for future work.

\section{REFERENCES}

[1] B. Rankov and A. Wittneben, "Achievable rate regions for the two-way relay channel," in IEEE Int. Symp. Inf. Theory, 2006, pp. 1668-1672.

[2] C. Schnurr, T. Oechtering, and S. Stanczak, "Achievable rates for the restricted half-duplex two-way relay channel," in Signals, Systems and Computers, 2007. ACSSC 2007. Conference Record of the Forty-First Asilomar Conference on, 2007, pp. 1468-1472.

[3] C. Schnurr, S. Stanczak, and T. Oechtering, "Coding theorems for the restricted half-duplex two-way relay channel with joint decoding," in IEEE Int. Symp. Inf. Theory, 2008, pp. 2688-2692.

[4] A. Avestimehr, S. Diggavi, and D. Tse, "Wireless network information flow: A deterministic approach," IEEE Trans. Inf. Theory, vol. 57, no. 4 pp. 1872-1905, Apr. 2011

[5] M. H. Yassaee and M. R. Aref, "Slepian-Wolf coding over cooperative relay networks," IEEE Trans. Inf. Theory, vol. 57, no. 6, pp. 3462-3482, Jun. 2011.

[6] S. Lim, Y. Kim, A. El Gamal, and S. Chung, "Noisy network coding," IEEE Trans. Inf. Theory, vol. 57, no. 5, pp. 3132-3152, May 2011.

[7] V. Nagpal, I. Wang, M. Jorgovanovic, D. Tse, and B. Nikolic, "Coding and system design for quantize-map-and-forward relaying," IEEE J. Sel. Areas Commun., vol. 31, no. 8, pp. 1423-1435, Aug. 2013.

[8] A. Sengupta, S. Brahma, A. Ozgur, C. Fragouli, and S. Diggavi, "Graph-based codes for quantize-map-and-forward relaying," in IEEE Inf. Theory Workshop, Oct. 2011, pp. 140-144.

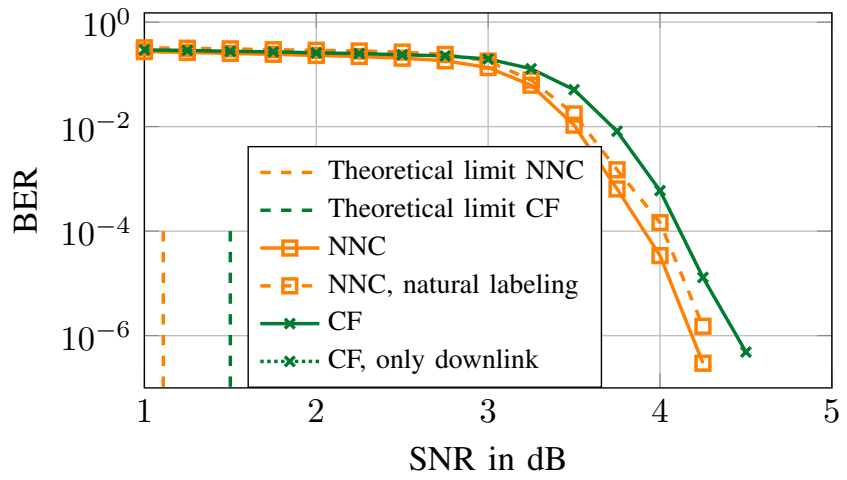

(a) Bit error rate for whole system.

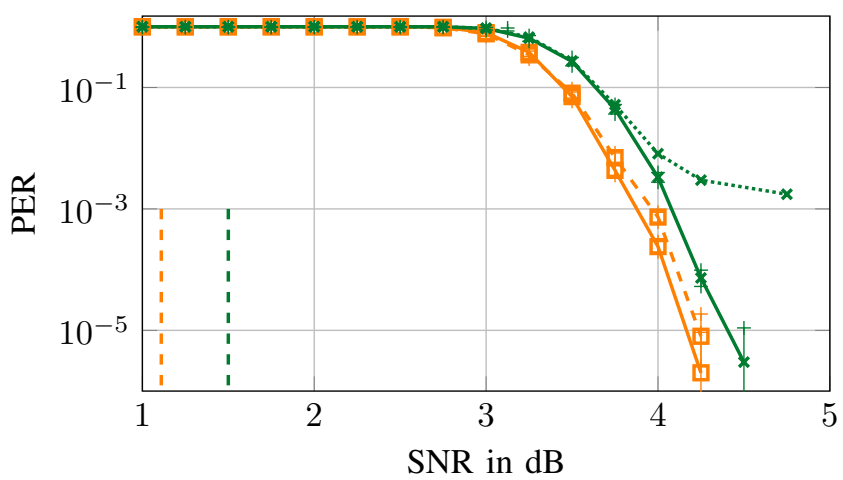

(b) Packet error rate for whole system.

Fig. 5: Bit error rate (BER) and packet error rate (PER). The SNR refers to $\frac{P_{1}}{N_{r}}=\frac{P_{2}}{N_{r}}$, the downlink SNR $\frac{P_{r}}{N_{2}}$ has an offset of $6 \mathrm{~dB}$.

[9] A. Sengupta, I.-H. Wang, and C. Fragouli, "Optimizing quantize-mapand-forward relaying for Gaussian diamond networks," in IEEE Inf. Theory Workshop, Sep. 2012, pp. 381-385.

[10] W. Zhong and J. Garcia-Frias, "LDGM codes for channel coding and joint source-channel coding of correlated sources," EURASIP J. Appl. Signal Process., vol. 2005, pp. 942-953, Jan. 2005.

[11] M. Heindlmaier, Network Coding for Two-Way Relay Channels and Broadcast Erasure Channels with Feedback. Dr. Hut Verlag, 2015.

[12] S. J. Kim, N. Devroye, P. Mitran, and V. Tarokh, "Achievable rate regions and performance comparison of half duplex bi-directional relaying protocols," IEEE Trans. Inf. Theory, vol. 57, no. 10, pp. 6405 -6418 , Oct. 2011.

[13] E. Tuncel, "Slepian-Wolf coding over broadcast channels," IEEE Trans. Inf. Theory, vol. 52, no. 4, pp. 1469-1482, Apr. 2006.

[14] S. Saeedi Bidokhti and V. M. Prabhakaran, "Is non-unique decoding necessary?" IEEE Trans. Inf. Theory, vol. 60, no. 5, May 2014.

[15] A. G. i Fàbregas, A. Martinez, and G. Caire, Bit-interleaved coded modulation. NOW Publishers, 2008.

[16] D. Divsalar, H. Jin, and R. J. McEliece, "Coding theorems for "turbolike" codes," in Allerton Conf. on Commun., Control, and Computing, vol. 36, 1998, pp. 201-210.

[17] H. Jin, A. Khandekar, and R. McEliece, "Irregular repeat-accumulate codes," in Proc. 2nd Int. Symp. Turbo codes, 2000, pp. 1-8.

[18] F. Gray, "Pulse code communication," U.S. Patent 2,632,058, 1953.

[19] X. Li and J. Ritcey, "Bit-interleaved coded modulation with iterative decoding," IEEE Commun. Lett., vol. 1, no. 6, pp. 169-171, Nov. 1997.

[20] T. Richardson and R. Urbanke, Modern Coding Theory. Cambridge University Press, 2008.

[21] F. Kschischang, B. Frey, and H.-A. Loeliger, "Factor graphs and the sum-product algorithm," IEEE Trans. Inf. Theory, vol. 47, no. 2, pp. 498-519, Feb. 2001. 